\title{
Nonlinear second-order impulsive $q$-difference Langevin equation with boundary conditions
}

\author{
Jessada Tariboon ${ }^{1 *}$ and Sotiris K Ntouyas ${ }^{2}$
}

*Correspondence:

jessadat@kmutnb.ac.th

${ }^{1}$ Nonlinear Dynamic Analysis

Research Center, Department of

Mathematics, Faculty of Applied

Science, King Mongkut's University

of Technology North Bangkok,

Bangkok, Thailand

Full list of author information is

available at the end of the article

\begin{abstract}
In this paper, we discuss the existence and uniqueness of solutions for Langevin impulsive $q$-difference equations with boundary conditions. Our study relies on Banach's and Schaefer's fixed point theorems. Illustrative examples are also presented. MSC: 26A33; 39A13; 34A37

Keywords: $q_{k}$-derivative; $q_{k}$-integral; impulsive $q_{k}$-difference equation; existence; uniqueness; Langevin equation
\end{abstract}

\section{Introduction and preliminaries}

In recent years, the boundary value problems of fractional order differential equations have emerged as an important area of research, since these problems have applications in various disciplines of science and engineering such as mechanics, electricity, chemistry, biology, economics, control theory, signal and image processing, polymer rheology, regular variation in thermodynamics, biophysics, aerodynamics, viscoelasticity and damping, electro-dynamics of complex medium, wave propagation, blood flow phenomena, etc. [15]. Many researchers have studied the existence theory for nonlinear fractional differential equations with a variety of boundary conditions, for instance, see the papers [6-18], and the references therein.

The Langevin equation (first formulated by Langevin in 1908) is found to be an effective tool to describe the evolution of physical phenomena in fluctuating environments [19]. For some new developments on the fractional Langevin equation, see, for example, [20-27].

Nowadays there is a significant increase of activities in the area of $q$-calculus due to its applications in various fields such as mathematics, mechanics, and physics. The book by Kac and Cheung [28] covers many of the fundamental aspects of the quantum calculus. A variety of new results can be found in the papers [29-41] and the references cited therein.

Impulsive differential equations serve as basic models to study the dynamics of processes that are subject to sudden changes in their states. Recent development in this field has been motivated by many applied problems, such as control theory, population dynamics and medicine. For some recent works on the theory of impulsive differential equations, we refer the interested reader to the monographs [42-44].

Recently in [45] the notions of $q_{k}$-derivative and $q_{k}$-integral on finite intervals were introduced. Let us recall here these notions. For a fixed $k \in \mathbb{N} \cup\{0\}$ let $J_{k}:=\left[t_{k}, t_{k+1}\right] \subset \mathbb{R}$ be 
an interval and $0<q_{k}<1$ be a constant. We define $q_{k}$-derivative of a function $f: J_{k} \rightarrow \mathbb{R}$ at a point $t \in J_{k}$ as follows.

Definition 1.1 Assume $f: J_{k} \rightarrow \mathbb{R}$ is a continuous function and let $t \in J_{k}$. Then the expression

$$
\begin{aligned}
& D_{q_{k}} f(t)=\frac{f(t)-f\left(q_{k} t+\left(1-q_{k}\right) t_{k}\right)}{\left(1-q_{k}\right)\left(t-t_{k}\right)}, \quad t \neq t_{k}, \\
& D_{q_{k}} f\left(t_{k}\right)=\lim _{t \rightarrow t_{k}} D_{q_{k}} f(t),
\end{aligned}
$$

is called the $q_{k}$-derivative of function $f$ at $t$.

We say that $f$ is $q_{k}$-differentiable on $J_{k}$ provided $D_{q_{k}} f(t)$ exists for all $t \in J_{k}$. Note that if $t_{k}=0$ and $q_{k}=q$ in (1.1), then $D_{q_{k}} f=D_{q} f$, where $D_{q}$ is the well-known $q$-derivative of the function $f(t)$ defined by

$$
D_{q} f(t)=\frac{f(t)-f(q t)}{(1-q) t}
$$

In addition, we should define the higher $q_{k}$-derivative of functions.

Definition 1.2 Let $f: J_{k} \rightarrow \mathbb{R}$ be a continuous function, we call the second-order $q_{k}$-derivative $D_{q_{k}}^{2} f$ provided $D_{q_{k}} f$ is $q_{k}$-differentiable on $J_{k}$ with $D_{q_{k}}^{2} f=D_{q_{k}}\left(D_{q_{k}} f\right): J_{k} \rightarrow \mathbb{R}$. Similarly, we define higher order $q_{k}$-derivative $D_{q_{k}}^{n}: J_{k} \rightarrow \mathbb{R}$.

The $q_{k}$-integral is defined as follows.

Definition 1.3 Assume $f: J_{k} \rightarrow \mathbb{R}$ is a continuous function. Then the $q_{k}$-integral is defined by

$$
\int_{t_{k}}^{t} f(s) d_{q_{k}} s=\left(1-q_{k}\right)\left(t-t_{k}\right) \sum_{n=0}^{\infty} q_{k}^{n} f\left(q_{k}^{n} t+\left(1-q_{k}^{n}\right) t_{k}\right)
$$

for $t \in J_{k}$. Moreover, if $a \in\left(t_{k}, t\right)$ then the definite $q_{k}$-integral is defined by

$$
\begin{aligned}
\int_{a}^{t} f(s) d_{q_{k}} s= & \int_{t_{k}}^{t} f(s) d_{q_{k}} s-\int_{t_{k}}^{a} f(s) d_{q_{k}} s \\
= & \left(1-q_{k}\right)\left(t-t_{k}\right) \sum_{n=0}^{\infty} q_{k}^{n} f\left(q_{k}^{n} t+\left(1-q_{k}^{n}\right) t_{k}\right) \\
& -\left(1-q_{k}\right)\left(a-t_{k}\right) \sum_{n=0}^{\infty} q_{k}^{n} f\left(q_{k}^{n} a+\left(1-q_{k}^{n}\right) t_{k}\right) .
\end{aligned}
$$

Note that if $t_{k}=0$ and $q_{k}=q$, then (1.3) reduces to $q$-integral of a function $f(t)$, defined by $\int_{0}^{t} f(s) d_{q} s=(1-q) t \sum_{n=0}^{\infty} q^{n} f\left(q^{n} t\right)$ for $t \in[0, \infty)$.

For the basic properties of the $q_{k}$-derivative and $q_{k}$-integral we refer to [45]. 
In this paper we combine all the above subjects and investigate the nonlinear secondorder impulsive $q_{k}$-difference Langevin equation with boundary conditions of the form

$$
\left\{\begin{array}{l}
D_{q_{k}}\left(D_{q_{k}}+\lambda\right) x(t)=f(t, x(t)), \quad t \in J, t \neq t_{k}, \\
\Delta x\left(t_{k}\right)=I_{k}\left(x\left(t_{k}\right)\right), \quad k=1,2, \ldots, m, \\
D_{q_{k}} x\left(t_{k}^{+}\right)-D_{q_{k-1}} x\left(t_{k}\right)=I_{k}^{*}\left(x\left(t_{k}\right)\right), \quad k=1,2, \ldots, m, \\
\alpha x(0)+\beta D_{q_{0}} x(0)=x(T), \quad \gamma x(0)+\eta D_{q_{0}} x(0)=D_{q_{m}} x(T),
\end{array}\right.
$$

where $0=t_{0}<t_{1}<t_{2}<\cdots<t_{k}<\cdots<t_{m}<t_{m+1}=T, f: J \times \mathbb{R} \rightarrow \mathbb{R}$ is a continuous function, $\lambda$ is a given constant, $I_{k}, I_{k}^{*} \in C(\mathbb{R}, \mathbb{R}), \Delta x\left(t_{k}\right)=x\left(t_{k}^{+}\right)-x\left(t_{k}\right)$ for $k=1,2, \ldots, m$, $x\left(t_{k}^{+}\right)=\lim _{h \rightarrow 0+} x\left(t_{k}+h\right), 0<q_{k}<1$ for $k=0,1,2, \ldots, m$, and $\alpha, \beta, \gamma, \eta$ are given constants.

The rest of this paper is organized as follows. In Section 2, we present a preliminary result which will be used in this paper. In Section 3, we will consider the existence results for problem (1.4) while in Section 4, we will give examples to illustrate our main results.

\section{An auxiliary lemma}

In this section, we present an auxiliary lemma which will be used throughout this paper. Let $J=[0, T], J_{0}=\left[t_{0}, t_{1}\right], J_{k}=\left(t_{k}, t_{k+1}\right]$ for $k=1,2, \ldots, m$.

Lemma 2.1 Let $\lambda T(\eta+\beta \lambda-\alpha) \neq(\alpha-1)(\eta-1)+\gamma(T-\beta)$. The unique solution of problem (1.4) is given by

$$
\begin{aligned}
x(t)= & \frac{\delta_{1}+\delta_{2} t}{\Omega}\left\{\sum_{k=1}^{m}\left(\int_{t_{k-1}}^{t_{k}} \int_{t_{k-1}}^{s} f(r, x(r)) d_{q_{k-1}} r d_{q_{k-1}} s-\lambda \int_{t_{k-1}}^{t_{k}} x(s) d_{q_{k-1}} s+I_{k}\left(x\left(t_{k}\right)\right)\right)\right. \\
& +\sum_{k=1}^{m}\left(\int_{t_{k-1}}^{t_{k}} f(s, x(s)) d_{q_{k-1}} s+I_{k}^{*}\left(x\left(t_{k}\right)\right)+\lambda I_{k}\left(x\left(t_{k}\right)\right)\right)\left(T-t_{k}\right) \\
& \left.+\int_{t_{m}}^{T} \int_{t_{m}}^{s} f(r, x(r)) d_{q_{m}} r d_{q_{m}} s-\lambda \int_{t_{m}}^{T} x(s) d_{q_{m}} s\right\} \\
& +\frac{\delta_{3}+\delta_{4} t}{\Omega}\left\{\sum_{k=1}^{m}\left(\int_{t_{k-1}}^{t_{k}} f(s, x(s)) d_{q_{k-1}} s+I_{k}^{*}\left(x\left(t_{k}\right)\right)+\lambda I_{k}\left(x\left(t_{k}\right)\right)\right)\right. \\
& \left.+\int_{t_{m}}^{T} f(s, x(s)) d_{q_{m}} s\right\} \\
& +\sum_{0<t_{k}<t}\left(\int_{t_{k-1}}^{t_{k}} \int_{t_{k-1}}^{s} f(r, x(r)) d_{q_{k-1}} r d_{q_{k-1}} s-\lambda \int_{t_{k-1}}^{t_{k}} x(s) d_{q_{k-1}} s+I_{k}\left(x\left(t_{k}\right)\right)\right) \\
& +\sum_{0<t_{k}<t}\left(\int_{t_{k-1}}^{t_{k}} f(s, x(s)) d_{q_{k-1}} s+I_{k}^{*}\left(x\left(t_{k}\right)\right)+\lambda I_{k}\left(x\left(t_{k}\right)\right)\right)\left(t-t_{k}\right) \\
& +\int_{t_{k}}^{t} \int_{t_{k}}^{s} f(r, x(r)) d_{q_{k}} r d_{q_{k}} s-\lambda \int_{t_{k}}^{t} x(s) d_{q_{k}} s,
\end{aligned}
$$

with $\sum_{0<0}(\cdot)=0$, where

$$
\begin{aligned}
& \Omega=(\alpha-1)(\eta-1)-\lambda T(\eta+\beta \lambda-\alpha)+\gamma(T-\beta), \\
& \delta_{1}=\eta-1+\beta \lambda, \quad \delta_{2}=\lambda(\eta+\beta \lambda-1-\alpha)+1-\gamma, \\
& \delta_{3}=T-\beta, \quad \delta_{4}=\alpha-1-\beta \lambda .
\end{aligned}
$$


Proof For $t \in J_{0}$ using $q_{0}$-integral for the first equation of (1.4), we get

$$
D_{q_{0}} x(t)=D_{q_{0}} x(0)+\lambda x(0)+\int_{0}^{t} f(s, x(s)) d_{q_{0}} s-\lambda x(t) .
$$

Setting $x(0)=A$ and $D_{q_{0}} x(0)=B$, we have

$$
D_{q_{0}} x(t)=\lambda A+B+\int_{0}^{t} f(s, x(s)) d_{q_{0}} s-\lambda x(t),
$$

which leads to

$$
D_{q_{0}} x\left(t_{1}\right)=\lambda A+B+\int_{0}^{t_{1}} f(s, x(s)) d_{q_{0}} s-\lambda x\left(t_{1}\right) .
$$

For $t \in J_{0}$ we obtain by $q_{0}$-integrating (2.2),

$$
x(t)=A+(\lambda A+B) t+\int_{0}^{t} \int_{0}^{s} f(r, x(r)) d_{q_{0}} r d_{q_{0}} s-\lambda \int_{0}^{t} x(s) d_{q_{0}} s .
$$

In particular, for $t=t_{1}$

$$
x\left(t_{1}\right)=A+(\lambda A+B) t_{1}+\int_{0}^{t_{1}} \int_{0}^{s} f(r, x(r)) d_{q_{0}} r d_{q_{0}} s-\lambda \int_{0}^{t_{1}} x(s) d_{q_{0}} s .
$$

For $t \in J_{1}=\left(t_{1}, t_{2}\right], q_{1}$-integrating (1.4), we have

$$
D_{q_{1}} x(t)=D_{q_{1}} x\left(t_{1}^{+}\right)+\lambda x\left(t_{1}^{+}\right)+\int_{t_{1}}^{t} f(s, x(s)) d_{q_{1}} s-\lambda x(t) .
$$

From the second impulsive equations of (1.4), we have

$$
\begin{aligned}
D_{q_{1}} x(t)= & \lambda A+B+\int_{0}^{t_{1}} f(s, x(s)) d_{q_{0}} s+I_{1}^{*}\left(x\left(t_{1}\right)\right) \\
& +\lambda I_{1}\left(x\left(t_{1}\right)\right)+\int_{t_{1}}^{t} f(s, x(s)) d_{q_{1}} s-\lambda x(t) .
\end{aligned}
$$

Applying $q_{1}$-integral to (2.5) for $t \in J_{1}$, we obtain

$$
\begin{aligned}
x(t)= & x\left(t_{1}^{+}\right)+\left[\lambda A+B+\int_{0}^{t_{1}} f(s, x(s)) d_{q_{0}} s+I_{1}^{*}\left(x\left(t_{1}\right)\right)+\lambda I_{1}\left(x\left(t_{1}\right)\right)\right]\left(t-t_{1}\right) \\
& +\int_{t_{1}}^{t} \int_{t_{1}}^{s} f(r, x(r)) d_{q_{1}} r d_{q_{1}} s-\lambda \int_{t_{1}}^{t} x(s) d_{q_{1}} s .
\end{aligned}
$$

Using the second impulsive equation of (1.4) with (2.4) and (2.6), one has

$$
\begin{aligned}
x(t)= & A+(\lambda A+B) t_{1}+\int_{0}^{t_{1}} \int_{0}^{s} f(r, x(r)) d_{q_{0}} r d_{q_{0}} s-\lambda \int_{0}^{t_{1}} x(s) d_{q_{0}} s+I_{1}\left(x\left(t_{1}\right)\right) \\
& +\left[\lambda A+B+\int_{0}^{t_{1}} f(s, x(s)) d_{q_{0}} s+I_{1}^{*}\left(x\left(t_{1}\right)\right)+\lambda I_{1}\left(x\left(t_{1}\right)\right)\right]\left(t-t_{1}\right)
\end{aligned}
$$




$$
\begin{aligned}
& +\int_{t_{1}}^{t} \int_{t_{1}}^{s} f(r, x(r)) d_{q_{1}} r d_{q_{1}} s-\lambda \int_{t_{1}}^{t} x(s) d_{q_{1}} s \\
& =A+(\lambda A+B) t+\int_{0}^{t_{1}} \int_{0}^{s} f(r, x(r)) d_{q_{0}} r d_{q_{0}} s-\lambda \int_{0}^{t_{1}} x(s) d_{q_{0}} s+I_{1}\left(x\left(t_{1}\right)\right) \\
& +\left[\int_{0}^{t_{1}} f(s, x(s)) d_{q_{0}} s+I_{1}^{*}\left(x\left(t_{1}\right)\right)+\lambda I_{1}\left(x\left(t_{1}\right)\right)\right]\left(t-t_{1}\right) \\
& +\int_{t_{1}}^{t} \int_{t_{1}}^{s} f(r, x(r)) d_{q_{1}} r d_{q_{1}} s-\lambda \int_{t_{1}}^{t} x(s) d_{q_{1}} s .
\end{aligned}
$$

Repeating the above process, for $t \in J$, we get

$$
\begin{aligned}
x(t)= & A+(\lambda A+B) t \\
& +\sum_{0<t_{k}<t}\left(\int_{t_{k-1}}^{t_{k}} \int_{t_{k-1}}^{s} f(r, x(r)) d_{q_{k-1}} r d_{q_{k-1}} s-\lambda \int_{t_{k-1}}^{t_{k}} x(s) d_{q_{k-1}} s+I_{k}\left(x\left(t_{k}\right)\right)\right) \\
& +\sum_{0<t_{k}<t}\left(\int_{t_{k-1}}^{t_{k}} f(s, x(s)) d_{q_{k-1}} s+I_{k}^{*}\left(x\left(t_{k}\right)\right)+\lambda I_{k}\left(x\left(t_{k}\right)\right)\right)\left(t-t_{k}\right) \\
& +\int_{t_{k}}^{t} \int_{t_{k}}^{s} f(r, x(r)) d_{q_{k}} r d_{q_{k}} s-\lambda \int_{t_{k}}^{t} x(s) d_{q_{k}} s .
\end{aligned}
$$

For $t=T$, we get

$$
\begin{aligned}
x(T)= & (1+\lambda T) A+B T \\
& +\sum_{k=1}^{m}\left(\int_{t_{k-1}}^{t_{k}} \int_{t_{k-1}}^{s} f(r, x(r)) d_{q_{k-1}} r d_{q_{k-1}} s-\lambda \int_{t_{k-1}}^{t_{k}} x(s) d_{q_{k-1}} s+I_{k}\left(x\left(t_{k}\right)\right)\right) \\
& +\sum_{k=1}^{m}\left(\int_{t_{k-1}}^{t_{k}} f(s, x(s)) d_{q_{k-1}} s+I_{k}^{*}\left(x\left(t_{k}\right)\right)+\lambda I_{k}\left(x\left(t_{k}\right)\right)\right)\left(T-t_{k}\right) \\
& +\int_{t_{m}}^{T} \int_{t_{m}}^{s} f(r, x(r)) d_{q_{m}} r d_{q_{m}} s-\lambda \int_{t_{m}}^{T} x(s) d_{q_{m}} s .
\end{aligned}
$$

It is easy to see that

$$
\begin{aligned}
D_{q_{k}} x(t)= & \lambda A+B \\
& +\sum_{0<t_{k}<t}\left(\int_{t_{k-1}}^{t_{k}} f(s, x(s)) d_{q_{k-1}} s+I_{k}^{*}\left(x\left(t_{k}\right)\right)+\lambda I_{k}\left(x\left(t_{k}\right)\right)\right) \\
& +\int_{t_{k}}^{t} f(s, x(s)) d_{q_{k}} s-\lambda x(t) .
\end{aligned}
$$

For $t=T$ and using $x(T)=\alpha A+\beta B$, we have

$$
\begin{aligned}
D_{q_{m}} x(T)= & \lambda A+B \\
& +\sum_{k=1}^{m}\left(\int_{t_{k-1}}^{t_{k}} f(s, x(s)) d_{q_{k-1}} s+I_{k}^{*}\left(x\left(t_{k}\right)\right)+\lambda I_{k}\left(x\left(t_{k}\right)\right)\right) \\
& +\int_{t_{m}}^{T} f(s, x(s)) d_{q_{m}} s-\lambda x(T)
\end{aligned}
$$




$$
\begin{aligned}
= & (1-\alpha) \lambda A+(1-\lambda \beta) B \\
& +\sum_{k=1}^{m}\left(\int_{t_{k-1}}^{t_{k}} f(s, x(s)) d_{q_{k-1}} s+I_{k}^{*}\left(x\left(t_{k}\right)\right)+\lambda I_{k}\left(x\left(t_{k}\right)\right)\right) \\
& +\int_{t_{m}}^{T} f(s, x(s)) d_{q_{m}} s .
\end{aligned}
$$

Applying the boundary conditions of (1.4) with (2.8) and (2.9), it follows that

$$
\begin{aligned}
A= & \frac{\eta+\lambda \beta-1}{\Omega}\left\{\sum _ { k = 1 } ^ { m } \left(\int_{t_{k-1}}^{t_{k}} \int_{t_{k-1}}^{s} f(r, x(r)) d_{q_{k-1}} r d_{q_{k-1}} s\right.\right. \\
& \left.-\lambda \int_{t_{k-1}}^{t_{k}} x(s) d_{q_{k-1}} s+I_{k}\left(x\left(t_{k}\right)\right)\right) \\
& +\sum_{k=1}^{m}\left(\int_{t_{k-1}}^{t_{k}} f(s, x(s)) d_{q_{k-1}} s+I_{k}^{*}\left(x\left(t_{k}\right)\right)+\lambda I_{k}\left(x\left(t_{k}\right)\right)\right)\left(T-t_{k}\right) \\
& \left.+\int_{t_{m}}^{T} \int_{t_{m}}^{s} f(r, x(r)) d_{q_{m}} r d_{q_{m}} s-\lambda \int_{t_{m}}^{T} x(s) d_{q_{m}} s\right\} \\
& -\frac{\beta-T}{\Omega}\left\{\sum_{k=1}^{m}\left(\int_{t_{k-1}}^{t_{k}} f(s, x(s)) d_{q_{k-1}} s+I_{k}^{*}\left(x\left(t_{k}\right)\right)+\lambda I_{k}\left(x\left(t_{k}\right)\right)\right)+\int_{t_{m}}^{T} f(s, x(s)) d_{q_{m}} s\right\}
\end{aligned}
$$

and

$$
\begin{aligned}
B= & \frac{\alpha-1-\lambda T}{\Omega}\left\{\sum_{k=1}^{m}\left(\int_{t_{k-1}}^{t_{k}} f(s, x(s)) d_{q_{k-1}} s+I_{k}^{*}\left(x\left(t_{k}\right)\right)+\lambda I_{k}\left(x\left(t_{k}\right)\right)\right)\right. \\
& \left.+\int_{t_{m}}^{T} f(s, x(s)) d_{q_{m}} s\right\} \\
& -\frac{\gamma-1+\alpha \lambda}{\Omega}\left\{\sum _ { k = 1 } ^ { m } \left(\int_{t_{k-1}}^{t_{k}} \int_{t_{k-1}}^{s} f(r, x(r)) d_{q_{k-1}} r d_{q_{k-1}} s\right.\right. \\
& \left.-\lambda \int_{t_{k-1}}^{t_{k}} x(s) d_{q_{k-1}} s+I_{k}\left(x\left(t_{k}\right)\right)\right) \\
& +\sum_{k=1}^{m}\left(\int_{t_{k-1}}^{t_{k}} f(s, x(s)) d_{q_{k-1}} s+I_{k}^{*}\left(x\left(t_{k}\right)\right)+\lambda I_{k}\left(x\left(t_{k}\right)\right)\right)\left(T-t_{k}\right) \\
& \left.+\int_{t_{m}}^{T} \int_{t_{m}}^{s} f(r, x(r)) d_{q_{m}} r d_{q_{m}} s-\lambda \int_{t_{m}}^{T} x(s) d_{q_{m}} s\right\} .
\end{aligned}
$$

Substituting the values of $A$ and $B$ into (2.7), we get (2.1) as required. The proof is completed.

\section{Main results}

Let $P C(J, \mathbb{R})=\left\{x: J \rightarrow \mathbb{R}: x(t)\right.$ is continuous everywhere except for some $t_{k}$ at which $x\left(t_{k}^{+}\right)$ and $x\left(t_{k}^{-}\right)$exist and $\left.x\left(t_{k}^{-}\right)=x\left(t_{k}\right), k=1,2, \ldots, m\right\} . P C(J, \mathbb{R})$ is a Banach space with the norm $\|x\|_{P C}=\sup \{|x(t)| ; t \in J\}$. 
From Lemma 2.1, we define an operator $\mathcal{S}: P C(J, \mathbb{R}) \rightarrow P C(J, \mathbb{R})$ by

$$
\begin{aligned}
(\mathcal{S} x)(t)= & \frac{\delta_{1}+\delta_{2} t}{\Omega}\left\{\sum _ { k = 1 } ^ { m } \left(\int_{t_{k-1}}^{t_{k}} \int_{t_{k-1}}^{s} f(r, x(r)) d_{q_{k-1}} r d_{q_{k-1}} s\right.\right. \\
& \left.-\lambda \int_{t_{k-1}}^{t_{k}} x(s) d_{q_{k-1}} s+I_{k}\left(x\left(t_{k}\right)\right)\right) \\
& +\sum_{k=1}^{m}\left(\int_{t_{k-1}}^{t_{k}} f(s, x(s)) d_{q_{k-1}} s+I_{k}^{*}\left(x\left(t_{k}\right)\right)+\lambda I_{k}\left(x\left(t_{k}\right)\right)\right)\left(T-t_{k}\right) \\
& \left.+\int_{t_{m}}^{T} \int_{t_{m}}^{s} f(r, x(r)) d_{q_{m}} r d_{q_{m}} s-\lambda \int_{t_{m}}^{T} x(s) d_{q_{m}} s\right\} \\
& +\frac{\delta_{3}+\delta_{4} t}{\Omega}\left\{\sum_{k=1}^{m}\left(\int_{t_{k-1}}^{t_{k}} f(s, x(s)) d_{q_{k-1}} s+I_{k}^{*}\left(x\left(t_{k}\right)\right)+\lambda I_{k}\left(x\left(t_{k}\right)\right)\right)\right. \\
& \left.+\int_{t_{m}}^{T} f(s, x(s)) d_{q_{m}} s\right\} \\
& +\sum_{0<t_{k}<t}\left(\int_{t_{k-1}}^{t_{k}} \int_{t_{k-1}}^{s} f(r, x(r)) d_{q_{k-1}} r d_{q_{k-1}} s-\lambda \int_{t_{k-1}}^{t_{k}} x(s) d_{q_{k-1}} s+I_{k}\left(x\left(t_{k}\right)\right)\right) \\
& +\sum_{0<t_{k}<t}\left(\int_{t_{k-1}}^{t_{k}} f(s, x(s)) d_{q_{k-1}} s+I_{k}^{*}\left(x\left(t_{k}\right)\right)+\lambda I_{k}\left(x\left(t_{k}\right)\right)\right)\left(t-t_{k}\right) \\
& +\int_{t_{k}}^{t} \int_{t_{k}}^{s} f(r, x(r)) d_{q_{k}} r d_{q_{k}} s-\lambda \int_{t_{k}}^{t} x(s) d_{q_{k}} s,
\end{aligned}
$$

where constants $\delta_{1}, \delta_{2}, \delta_{3}, \delta_{4}$, and $\Omega$ are defined as in Lemma 2.1. It should be noticed that problem (1.4) has solutions if and only if the operator $\mathcal{S}$ has fixed points.

Our first result is an existence and uniqueness result for the impulsive boundary value problem (1.4) by using the Banach contraction mapping principle.

For convenience, we set

$$
\begin{aligned}
\Lambda_{1}= & \left(\frac{\left|\delta_{1}\right|+\left|\delta_{2}\right| T+|\Omega|}{|\Omega|}\right)\left[L_{1} \sum_{k=1}^{m+1} \frac{\left(t_{k}-t_{k-1}\right)^{2}}{1+q_{k-1}}+|\lambda| \sum_{k=1}^{m+1}\left(t_{k}-t_{k-1}\right)\right. \\
& \left.+m L_{2}+\sum_{k=1}^{m}\left(L_{1}\left(t_{k}-t_{k-1}\right)+L_{3}+|\lambda| L_{2}\right)\left(T-t_{k}\right)\right] \\
& +\left(\frac{\left|\delta_{3}\right|+\left|\delta_{4}\right| T}{|\Omega|}\right)\left[L_{1} T+m L_{3}+m|\lambda| L_{2}\right]
\end{aligned}
$$

and

$$
\begin{aligned}
\Lambda_{2}= & \left(\frac{\left|\delta_{1}\right|+\left|\delta_{2}\right| T+|\Omega|}{|\Omega|}\right)\left[K_{1} \sum_{k=1}^{m+1} \frac{\left(t_{k}-t_{k-1}\right)^{2}}{1+q_{k-1}}+m K_{2}\right. \\
& \left.+\sum_{k=1}^{m}\left(K_{1}\left(t_{k}-t_{k-1}\right)+K_{3}+|\lambda| K_{2}\right)\left(T-t_{k}\right)\right] \\
& +\left(\frac{\left|\delta_{3}\right|+\left|\delta_{4}\right| T}{|\Omega|}\right)\left[K_{1} T+m K_{3}+m|\lambda| K_{2}\right] .
\end{aligned}
$$


Theorem 3.1 Assume that the following conditions hold:

$\left(\mathrm{H}_{1}\right) f:[0, T] \times \mathbb{R} \rightarrow \mathbb{R}$ is a continuous function and there exists a constant $L_{1}>0$ such that

$$
|f(t, x)-f(t, y)| \leq L_{1}|x-y|
$$

for each $t \in J$ and $x, y \in \mathbb{R}$.

$\left(\mathrm{H}_{2}\right)$ The functions $I_{k}, I_{k}^{*}: \mathbb{R} \rightarrow \mathbb{R}$ are continuous and there exist constants $L_{2}, L_{3}>0$ such that

$$
\left|I_{k}(x)-I_{k}(y)\right| \leq L_{2}|x-y| \text { and }\left|I_{k}^{*}(x)-I_{k}^{*}(y)\right| \leq L_{3}|x-y|
$$

for each $x, y \in \mathbb{R}, k=1,2, \ldots, m$.

If

$$
\Lambda_{1} \leq \delta<1,
$$

where $\Lambda_{1}$ is defined by (3.2), then the impulsive $q_{k}$-difference Langevin boundary value problem (1.4) has a unique solution on $J$.

Proof Firstly, we transform the impulsive $q_{k}$-difference Langevin boundary value problem (1.4) into a fixed point problem, $x=\mathcal{S} x$, where the operator $\mathcal{S}$ is defined by (3.1). Applying the Banach contraction mapping principle, we shall show that $\mathcal{S}$ has a fixed point which is the unique solution of the boundary value problem (1.4).

Let $K_{1}, K_{2}$, and $K_{3}$ be nonnegative constants such that $K_{1}=\sup _{t \in J}|f(t, 0)|, K_{2}=$ $\sup \left\{\left|I_{k}(0)\right|: k=1,2, \ldots, m\right\}$, and $K_{3}=\sup \left\{\left|I_{k}^{*}(0)\right|: k=1,2, \ldots, m\right\}$. We choose a suitable constant $\rho$ by

$$
\rho \geq \frac{\Lambda_{2}}{1-\varepsilon}
$$

where $\delta \leq \varepsilon<1$ and $\Lambda_{2}$ defined by (3.3). Now, we will show that $\mathcal{S} B_{\rho} \subset B_{\rho}$, where a set $B_{\rho}$ is defined as $B_{\rho}=\{x \in P C(J, \mathbb{R}):\|x\| \leq \rho\}$. For $x \in B_{\rho}$, we have

$$
\begin{aligned}
\|\mathcal{S} x\| \leq \sup _{t \in J} & \left\{\frac { | \delta _ { 1 } | + | \delta _ { 2 } | t } { | \Omega | } \left\{\sum _ { k = 1 } ^ { m } \left(\int_{t_{k-1}}^{t_{k}} \int_{t_{k-1}}^{s}|f(r, x(r))| d_{q_{k-1}} r d_{q_{k-1}} s\right.\right.\right. \\
& \left.+|\lambda| \int_{t_{k-1}}^{t_{k}}|x(s)| d_{q_{k-1}} s+\left|I_{k}\left(x\left(t_{k}\right)\right)\right|\right) \\
& +\sum_{k=1}^{m}\left(\int_{t_{k-1}}^{t_{k}}|f(s, x(s))| d_{q_{k-1}} s+\left|I_{k}^{*}\left(x\left(t_{k}\right)\right)\right|+|\lambda|\left|I_{k}\left(x\left(t_{k}\right)\right)\right|\right)\left(T-t_{k}\right) \\
& \left.+\int_{t_{m}}^{T} \int_{t_{m}}^{s}|f(r, x(r))| d_{q_{m}} r d_{q_{m}} s+|\lambda| \int_{t_{m}}^{T}|x(s)| d_{q_{m}} s\right\} \\
& +\frac{\left|\delta_{3}\right|+\left|\delta_{4}\right| t}{|\Omega|}\left\{\sum_{k=1}^{m}\left(\int_{t_{k-1}}^{t_{k}}|f(s, x(s))| d_{q_{k-1}} s+\left|I_{k}^{*}\left(x\left(t_{k}\right)\right)\right|+|\lambda|\left|I_{k}\left(x\left(t_{k}\right)\right)\right|\right)\right. \\
& \left.+\int_{t_{m}}^{T}|f(s, x(s))| d_{q_{m}} s\right\}
\end{aligned}
$$


Tariboon and Ntouyas Boundary Value Problems 2014, 2014:85

Page 9 of 19

http://www.boundaryvalueproblems.com/content/2014/1/85

$$
\begin{aligned}
& +\sum_{0<t_{k}<t}\left(\int_{t_{k-1}}^{t_{k}} \int_{t_{k-1}}^{s}|f(r, x(r))| d_{q_{k-1}} r d_{q_{k-1}} s+|\lambda| \int_{t_{k-1}}^{t_{k}}|x(s)| d_{q_{k-1}} s+\left|I_{k}\left(x\left(t_{k}\right)\right)\right|\right) \\
& +\sum_{0<t_{k}<t}\left(\int_{t_{k-1}}^{t_{k}}|f(s, x(s))| d_{q_{k-1}} s+\left|I_{k}^{*}\left(x\left(t_{k}\right)\right)\right|+|\lambda|\left|I_{k}\left(x\left(t_{k}\right)\right)\right|\right)\left(t-t_{k}\right) \\
& \left.+\int_{t_{k}}^{t} \int_{t_{k}}^{s}|f(r, x(r))| d_{q_{k}} r d_{q_{k}} s+|\lambda| \int_{t_{k}}^{t}|x(s)| d_{q_{k}} s\right\} \\
& \leq \frac{\left|\delta_{1}\right|+\left|\delta_{2}\right| T}{|\Omega|}\left\{\sum _ { k = 1 } ^ { m } \left(\int_{t_{k-1}}^{t_{k}} \int_{t_{k-1}}^{s}(|f(r, x(r))-f(r, 0)|+|f(r, 0)|) d_{q_{k-1}} r d_{q_{k-1}} s\right.\right. \\
& \left.+|\lambda| \int_{t_{k-1}}^{t_{k}}\|x\| d_{q_{k-1}} s+\left(\left|I_{k}\left(x\left(t_{k}\right)\right)-I_{k}(0)\right|+\left|I_{k}(0)\right|\right)\right) \\
& +\sum_{k=1}^{m}\left(\int_{t_{k-1}}^{t_{k}}(|f(s, x(s))-f(s, 0)|+|f(s, 0)|) d_{q_{k-1}} s\right. \\
& +\left(\left|I_{k}^{*}\left(x\left(t_{k}\right)\right)-I_{k}^{*}(0)\right|+\left|I_{k}^{*}(0)\right|\right) \\
& \left.+|\lambda|\left(\left|I_{k}\left(x\left(t_{k}\right)\right)-I_{k}(x(0))\right|+\left|I_{k}(x(0))\right|\right)\right)\left(T-t_{k}\right) \\
& \left.+\int_{t_{m}}^{T} \int_{t_{m}}^{s}(|f(r, x(r))-f(r, 0)|+|f(r, 0)|) d_{q_{m}} r d_{q_{m}} s+|\lambda| \int_{t_{m}}^{T}\|x\| d_{q_{m}} s\right\} \\
& +\frac{\left|\delta_{3}\right|+\left|\delta_{4}\right| T}{|\Omega|}\left\{\sum _ { k = 1 } ^ { m } \left(\int_{t_{k-1}}^{t_{k}}(|f(s, x(s))-f(s, 0)|+|f(s, 0)|) d_{q_{k-1}} s\right.\right. \\
& \left.+\left(\left|I_{k}^{*}\left(x\left(t_{k}\right)\right)-I_{k}^{*}(0)\right|+\left|I_{k}^{*}(0)\right|\right)+|\lambda|\left(\left|I_{k}\left(x\left(t_{k}\right)\right)-I_{k}(x(0))\right|+\left|I_{k}(x(0))\right|\right)\right) \\
& \left.+\int_{t_{m}}^{T}(|f(s, x(s))-f(s, 0)|+|f(s, 0)|) d_{q_{m}} s\right\} \\
& +\sum_{k=1}^{m}\left(\int_{t_{k-1}}^{t_{k}} \int_{t_{k-1}}^{s}(|f(r, x(r))-f(r, 0)|+|f(r, 0)|) d_{q_{k-1}} r d_{q_{k-1}} s\right. \\
& \left.+|\lambda| \int_{t_{k-1}}^{t_{k}}\|x\| d_{q_{k-1}} s+\left(\left|I_{k}\left(x\left(t_{k}\right)\right)-I_{k}(x(0))\right|+\left|I_{k}(x(0))\right|\right)\right) \\
& +\sum_{k=1}^{m}\left(\int_{t_{k-1}}^{t_{k}}(|f(s, x(s))-f(s, 0)|+|f(s, 0)|) d_{q_{k-1}} s\right. \\
& +\left(\left|I_{k}^{*}\left(x\left(t_{k}\right)\right)-I_{k}^{*}(0)\right|+\left|I_{k}^{*}(0)\right|\right) \\
& \left.+|\lambda|\left(\left|I_{k}\left(x\left(t_{k}\right)\right)-I_{k}(x(0))\right|+\left|I_{k}(x(0))\right|\right)\right)\left(T-t_{k}\right) \\
& +\int_{t_{m}}^{T} \int_{t_{m}}^{s}(|f(r, x(r))-f(r, 0)|+|f(r, 0)|) d_{q_{m}} r d_{q_{m}} s+|\lambda| \int_{t_{m}}^{T}\|x\| d_{q_{m}} s \\
& \leq \frac{\left|\delta_{1}\right|+\left|\delta_{2}\right| T}{|\Omega|}\left\{\sum _ { k = 1 } ^ { m } \left(\int_{t_{k-1}}^{t_{k}} \int_{t_{k-1}}^{s}\left(L_{1} \rho+K_{1}\right) d_{q_{k-1}} r d_{q_{k-1}} s+|\lambda| \int_{t_{k-1}}^{t_{k}} \rho d_{q_{k-1}} s\right.\right. \\
& \left.+\left(L_{2} \rho+K_{2}\right)\right)+\sum_{k=1}^{m}\left(\int_{t_{k-1}}^{t_{k}}\left(L_{1} \rho+K_{1}\right) d_{q_{k-1}} s+\left(L_{3} \rho+K_{3}\right)\right.
\end{aligned}
$$


Tariboon and Ntouyas Boundary Value Problems 2014, 2014:85

Page 10 of 19

http://www.boundaryvalueproblems.com/content/2014/1/85

$$
\begin{aligned}
& \left.\left.+|\lambda|\left(L_{2} \rho+K_{2}\right)\right)\left(T-t_{k}\right)+\int_{t_{m}}^{T} \int_{t_{m}}^{s}\left(L_{1} \rho+K_{1}\right) d_{q_{m}} r d_{q_{m}} s+|\lambda| \int_{t_{m}}^{T} \rho d_{q_{m}} s\right\} \\
& +\frac{\left|\delta_{3}\right|+\left|\delta_{4}\right| T}{|\Omega|}\left\{\sum_{k=1}^{m}\left(\int_{t_{k-1}}^{t_{k}}\left(L_{1} \rho+K_{1}\right) d_{q_{k-1}} s+\left(L_{3} \rho+K_{3}\right)+|\lambda|\left(L_{2} \rho+K_{2}\right)\right)\right. \\
& \left.+\int_{t_{m}}^{T}\left(L_{1} \rho+K_{1}\right) d_{q_{m}} s\right\}+\sum_{k=1}^{m}\left(\int_{t_{k-1}}^{t_{k}} \int_{t_{k-1}}^{s}\left(L_{1} \rho+K_{1}\right) d_{q_{k-1}} r d_{q_{k-1}} s\right. \\
& \left.+|\lambda| \int_{t_{k-1}}^{t_{k}} \rho d_{q_{k-1}} s+\left(L_{2} \rho+K_{2}\right)\right)+\sum_{k=1}^{m}\left(\int_{t_{k-1}}^{t_{k}}\left(L_{1} \rho+K_{1}\right) d_{q_{k-1}} s\right. \\
& \left.+\left(L_{3} \rho+K_{3}\right)+|\lambda|\left(L_{2} \rho+K_{2}\right)\right)\left(T-t_{k}\right) \\
& +\int_{t_{m}}^{T} \int_{t_{m}}^{s}\left(L_{1} \rho+K_{1}\right) d_{q_{m}} r d_{q_{m}} s+|\lambda| \int_{t_{m}}^{T} \rho d_{q_{m}} s \\
& =\Lambda_{1} \rho+\Lambda_{2} \leq(\delta+1-\varepsilon) \rho \leq \rho,
\end{aligned}
$$

which implies that $\mathcal{S} B_{\rho} \subset B_{\rho}$.

For any $x, y \in P C(J, \mathbb{R})$ and for each $t \in J$, we have

$$
\begin{aligned}
\mid \mathcal{S} x( & t)-\mathcal{S} y(t) \mid \\
\leq & \frac{\left|\delta_{1}\right|+\left|\delta_{2}\right| t}{|\Omega|}\left\{\sum _ { k = 1 } ^ { m } \left(\int_{t_{k-1}}^{t_{k}} \int_{t_{k-1}}^{s}|f(r, x(r))-f(r, y(r))| d_{q_{k-1}} r d_{q_{k-1}} s\right.\right. \\
& \left.+|\lambda| \int_{t_{k-1}}^{t_{k}}|x(s)-y(s)| d_{q_{k-1}} s+\left|I_{k}\left(x\left(t_{k}\right)\right)-I_{k}\left(y\left(t_{k}\right)\right)\right|\right) \\
& +\sum_{k=1}^{m}\left(\int_{t_{k-1}}^{t_{k}}|f(s, x(s))-f(s, y(s))| d_{q_{k-1}} s\right. \\
& \left.+\left|I_{k}^{*}\left(x\left(t_{k}\right)\right)-I_{k}^{*}\left(y\left(t_{k}\right)\right)\right|+|\lambda|\left|I_{k}\left(x\left(t_{k}\right)\right)-I_{k}\left(y\left(t_{k}\right)\right)\right|\right)\left(T-t_{k}\right) \\
& \left.+\int_{t_{m}}^{T} \int_{t_{m}}^{s}|f(r, x(r))-f(r, y(r))| d_{q_{m}} r d_{q_{m}} s+|\lambda| \int_{t_{m}}^{T}|x(s)-y(s)| d_{q_{m}} s\right\} \\
& +\frac{\left|\delta_{3}\right|+\left|\delta_{4}\right| t}{|\Omega|}\left\{\sum _ { k = 1 } ^ { m } \left(\int_{t_{k-1}}^{t_{k}}|f(s, x(s))-f(s, y(s))| d_{q_{k-1}} s\right.\right. \\
& +\sum_{0<t_{k}<t}\left(\int_{t_{k-1}}^{t_{k}} \int_{t_{k-1}}^{t_{k}}|f(r, x(r))-f(r, y(r))| d_{q_{k-1}} r d_{q_{k-1}} s\right. \\
& \left.+\left|I_{k-1}^{*}\left(x\left(t_{k}\right)\right)-I_{k}^{*}\left(y\left(t_{k}\right)\right)\right|+|\lambda|\left|I_{k}\left(x\left(t_{k}\right)\right)-I_{k}\left(y\left(t_{k}\right)\right)\right|\right) \\
& \left.+\int_{t_{m}}^{T}|f(s, x(s))-f(s, y(s))| d_{q_{m}} s\right\} \\
& \left.+t_{k-1}\right\}
\end{aligned}
$$




$$
\begin{aligned}
& \left.+|\lambda|\left|I_{k}\left(x\left(t_{k}\right)\right)-I_{k}\left(y\left(t_{k}\right)\right)\right|\right)\left(t-t_{k}\right)+\int_{t_{k}}^{t} \int_{t_{k}}^{s}|f(r, x(r))-f(r, y(r))| d_{q_{k}} r d_{q_{k}} s \\
& +|\lambda| \int_{t_{k}}^{t}|x(s)-y(s)| d_{q_{k}} s \\
& \leq \frac{\left|\delta_{1}\right|+\left|\delta_{2}\right| T}{|\Omega|}\|x-y\|\left\{\sum_{k=1}^{m}\left(L_{1} \frac{\left(t_{k}-t_{k-1}\right)^{2}}{1+q_{k-1}}+|\lambda|\left(t_{k}-t_{k-1}\right)+L_{2}\right)\right. \\
& \left.+\sum_{k=1}^{m}\left(L_{1}\left(t_{k}-t_{k-1}\right)+L_{3}+|\lambda| L_{2}\right)\left(T-t_{k}\right)+L_{1} \frac{\left(T-t_{m}\right)^{2}}{1+q_{m}}+|\lambda|\left(T-t_{m}\right)\right\} \\
& +\frac{\left|\delta_{3}\right|+\left|\delta_{4}\right| T}{|\Omega|}\|x-y\|\left\{\sum_{k=1}^{m}\left(L_{1}\left(t_{k}-t_{k-1}\right)+L_{3}+|\lambda| L_{2}\right)+L_{1}\left(T-t_{m}\right)\right\} \\
& +\|x-y\| \sum_{k=1}^{m}\left(L_{1} \frac{\left(t_{k}-t_{k-1}\right)^{2}}{1+q_{k-1}}+|\lambda|\left(t_{k}-t_{k-1}\right)+L_{2}\right) \\
& +\|x-y\| \sum_{k=1}^{m}\left(L_{1}\left(t_{k}-t_{k-1}\right)+L_{3}+|\lambda| L_{2}\right)\left(T-t_{k}\right)+L_{1} \frac{\left(T-t_{m}\right)^{2}}{1+q_{m}}\|x-y\| \\
& +|\lambda|\left(T-t_{m}\right) \| x-y|| \\
& =\Lambda_{1}\|x-y\|,
\end{aligned}
$$

which implies that $\|\mathcal{S} x-\mathcal{S} y\| \leq \Lambda_{1}\|x-y\|$. As $\Lambda_{1}<1, \mathcal{S}$ is a contraction. Therefore, by the Banach contraction mapping principle, we find that $\mathcal{S}$ has a fixed point which is the unique solution of problem (1.4).

The second existence result is based on Schaefer's fixed point theorem.

Theorem 3.2 Assume that the following conditions hold:

$\left(\mathrm{H}_{3}\right) f: J \times \mathbb{R} \rightarrow \mathbb{R}$ is a continuous function and there exists a constant $M_{1}>0$ such that

$$
|f(t, x)| \leq M_{1}
$$

for each $t \in J$ and all $x \in \mathbb{R}$.

$\left(\mathrm{H}_{4}\right)$ The functions $I_{k}, I_{k}^{*}: \mathbb{R} \rightarrow \mathbb{R}$ are continuous and there exist constants $M_{2}, M_{3}>0$ such that

$$
\left|I_{k}(x)\right| \leq M_{2} \quad \text { and } \quad\left|I_{k}^{*}(x)\right| \leq M_{3}
$$

for all $x \in \mathbb{R}, k=1,2, \ldots, m$.

If

$$
\frac{\left|\delta_{1}\right|+\left|\delta_{2}\right| T+|\Omega|}{|\Omega|}|\lambda| T<1
$$

then the impulsive $q_{k}$-difference Langevin boundary value problem (1.4) has at least one solution on $J$. 
Proof We shall use Schaefer's fixed point theorem to prove that the operator $\mathcal{S}$ defined by (3.1) has a fixed point. We divide the proof into four steps.

Step 1: Continuity of $\mathcal{S}$.

Let $\left\{x_{n}\right\}$ be a sequence such that $x_{n} \rightarrow x$ in $P C(J, \mathbb{R})$. Since $f$ is a continuous function on $J \times \mathbb{R}$ and $I_{k}, I_{k}^{*}$ are continuous functions on $\mathbb{R}$ for $k=1,2, \ldots, m$, we have

$$
f\left(t, x_{n}(t)\right) \rightarrow f(t, x(t)), \quad I_{k}\left(x_{n}\left(t_{k}\right)\right) \rightarrow I_{k}\left(x\left(t_{k}\right)\right) \quad \text { and } \quad I_{k}^{*}\left(x_{n}\left(t_{k}\right)\right) \rightarrow I_{k}^{*}\left(x\left(t_{k}\right)\right)
$$

for $k=1,2, \ldots, m$, as $n \rightarrow \infty$.

Then, for each $t \in J$, we get

$$
\begin{aligned}
& \left|\left(\mathcal{S} x_{n}\right)(t)-(\mathcal{S} x)(t)\right| \\
& \leq \frac{\left|\delta_{1}\right|+\left|\delta_{2}\right| t}{|\Omega|}\left\{\sum _ { k = 1 } ^ { m } \left(\int_{t_{k-1}}^{t_{k}} \int_{t_{k-1}}^{s}\left|f\left(r, x_{n}(r)\right)-f(r, x(r))\right| d_{q_{k-1}} r d_{q_{k-1}} s\right.\right. \\
& \left.+\lambda \int_{t_{k-1}}^{t_{k}}\left|x_{n}(s)-x(s)\right| d_{q_{k-1}} s+\left|I_{k}\left(x_{n}\left(t_{k}\right)\right)-I_{k}\left(x\left(t_{k}\right)\right)\right|\right) \\
& +\sum_{k=1}^{m}\left(\int_{t_{k-1}}^{t_{k}}\left|f\left(s, x_{n}(s)\right)-f(s, x(s))\right| d_{q_{k-1}} s+\left|I_{k}^{*}\left(x_{n}\left(t_{k}\right)\right)-I_{k}^{*}\left(x\left(t_{k}\right)\right)\right|\right. \\
& \left.+|\lambda|\left|I_{k}\left(x_{n}\left(t_{k}\right)\right)-I_{k}\left(x\left(t_{k}\right)\right)\right|\right)\left(T-t_{k}\right) \\
& \left.+\int_{t_{m}}^{T} \int_{t_{m}}^{s}\left|f\left(r, x_{n}(r)\right)-f(r, x(r))\right| d_{q_{m}} r d_{q_{m}} s+|\lambda| \int_{t_{m}}^{T}\left|x_{n}(s)-x(s)\right| d_{q_{m}} s\right\} \\
& +\frac{\left|\delta_{3}\right|+\left|\delta_{4}\right| t}{\Omega}\left\{\sum _ { k = 1 } ^ { m } \left(\int_{t_{k-1}}^{t_{k}}\left|f\left(s, x_{n}(s)\right)-f(s, x(s))\right| d_{q_{k-1}} s\right.\right. \\
& +\left|I_{k}^{*}\left(x_{n}\left(t_{k}\right)\right)-I_{k}^{*}\left(x\left(t_{k}\right)\right)\right| \\
& \left.\left.+|\lambda|\left|I_{k}\left(x_{n}\left(t_{k}\right)\right)-I_{k}\left(x\left(t_{k}\right)\right)\right|\right)+\int_{t_{m}}^{T}\left|f\left(s, x_{n}(s)\right)-f(s, x(s))\right| d_{q_{m}} s\right\} \\
& +\sum_{0<t_{k}<t}\left(\int_{t_{k-1}}^{t_{k}} \int_{t_{k-1}}^{s}\left|f\left(r, x_{n}(r)\right)-f(r, x(r))\right| d_{q_{k-1}} r d_{q_{k-1}} s\right. \\
& \left.+|\lambda| \int_{t_{k-1}}^{t_{k}}\left|x_{n}(s)-x(s)\right| d_{q_{k-1}} s+\left|I_{k}\left(x_{n}\left(t_{k}\right)\right)-I_{k}\left(x\left(t_{k}\right)\right)\right|\right) \\
& +\sum_{0<t_{k}<t}\left(\int_{t_{k-1}}^{t_{k}}\left|f\left(s, x_{n}(s)\right)-f(s, x(s))\right| d_{q_{k-1}} s+\left|I_{k}^{*}\left(x_{n}\left(t_{k}\right)\right)-I_{k}^{*}\left(x\left(t_{k}\right)\right)\right|\right. \\
& \left.+|\lambda|\left|I_{k}\left(x_{n}\left(t_{k}\right)\right)-I_{k}\left(x\left(t_{k}\right)\right)\right|\right)\left(t-t_{k}\right) \\
& +\int_{t_{k}}^{t} \int_{t_{k}}^{s}\left|f\left(r, x_{n}(r)\right)-f(r, x(r))\right| d_{q_{k}} r d_{q_{k}} s+|\lambda| \int_{t_{k}}^{t}\left|x_{n}(s)-x(s)\right| d_{q_{k}} s,
\end{aligned}
$$

which gives $\left\|\mathcal{S} x_{n}-\mathcal{S} x\right\| \rightarrow 0$ as $n \rightarrow \infty$. This means that $\mathcal{S}$ is continuous.

Step 2: $\mathcal{S}$ maps bounded sets into bounded sets in $P C(J, \mathbb{R})$. 
Let us prove that for any $\rho^{*}>0$, there exists a positive constant $\sigma$ such that for each $x \in B_{\rho^{*}}=\left\{x \in P C(J, \mathbb{R}):\|x\| \leq \rho^{*}\right\}$, we have $\|\mathcal{S} x\| \leq \sigma$. For any $x \in B_{\rho^{*}}$, we have

$|(\mathcal{S} x)(t)|$

$$
\begin{aligned}
& \leq \frac{\left|\delta_{1}\right|+\left|\delta_{2}\right| T}{|\Omega|}\left\{\sum _ { k = 1 } ^ { m } \left(\int_{t_{k-1}}^{t_{k}} \int_{t_{k-1}}^{s}|f(r, x(r))| d_{q_{k-1}} r d_{q_{k-1}} s+|\lambda| \int_{t_{k-1}}^{t_{k}}|x(s)| d_{q_{k-1}} s\right.\right. \\
& \left.+\left|I_{k}\left(x\left(t_{k}\right)\right)\right|\right)+\sum_{k=1}^{m}\left(\int_{t_{k-1}}^{t_{k}}|f(s, x(s))| d_{q_{k-1}} s+\left|I_{k}^{*}\left(x\left(t_{k}\right)\right)\right|\right. \\
& \left.\left.+|\lambda|\left|I_{k}\left(x\left(t_{k}\right)\right)\right|\right)\left(T-t_{k}\right)+\int_{t_{m}}^{T} \int_{t_{m}}^{s}|f(r, x(r))| d_{q_{m}} r d_{q_{m}} s+|\lambda| \int_{t_{m}}^{T}|x(s)| d_{q_{m}} s\right\} \\
& +\frac{\left|\delta_{3}\right|+\left|\delta_{4}\right| T}{|\Omega|}\left\{\sum _ { k = 1 } ^ { m } \left(\int_{t_{k-1}}^{t_{k}}|f(s, x(s))| d_{q_{k-1}} s\right.\right. \\
& \left.\left.+\left|I_{k}^{*}\left(x\left(t_{k}\right)\right)\right|+|\lambda|\left|I_{k}\left(x\left(t_{k}\right)\right)\right|\right)+\int_{t_{m}}^{T}|f(s, x(s))| d_{q_{m}} s\right\} \\
& +\sum_{k=1}^{m}\left(\int_{t_{k-1}}^{t_{k}} \int_{t_{k-1}}^{s}|f(r, x(r))| d_{q_{k-1}} r d_{q_{k-1}} s+|\lambda| \int_{t_{k-1}}^{t_{k}}|x(s)| d_{q_{k-1}} s+\left|I_{k}\left(x\left(t_{k}\right)\right)\right|\right) \\
& +\sum_{k=1}^{m}\left(\int_{t_{k-1}}^{t_{k}}|f(s, x(s))| d_{q_{k-1}} s+\left|I_{k}^{*}\left(x\left(t_{k}\right)\right)\right|+|\lambda|\left|I_{k}\left(x\left(t_{k}\right)\right)\right|\right)\left(T-t_{k}\right) \\
& +\int_{t_{m}}^{T} \int_{t_{m}}^{s}|f(r, x(r))| d_{q_{m}} r d_{q_{m}} s+|\lambda| \int_{t_{m}}^{T}|x(s)| d_{q_{m}} s \\
& \leq \frac{\left|\delta_{1}\right|+\left|\delta_{2}\right| T}{|\Omega|}\left\{\sum_{k=1}^{m}\left(M_{1} \frac{\left(t_{k}-t_{k-1}\right)^{2}}{1+q_{k-1}}+\rho^{*}|\lambda|\left(t_{k}-t_{k-1}\right)+M_{2}\right)\right. \\
& \left.+\sum_{k=1}^{m}\left(M_{1}\left(t_{k}-t_{k-1}\right)+M_{3}+|\lambda| M_{2}\right)\left(T-t_{k}\right)+M_{1} \frac{\left(T-t_{m}\right)^{2}}{1+q_{m}}+\rho^{*}|\lambda|\left(T-t_{m}\right)\right\} \\
& +\frac{\left|\delta_{3}\right|+\left|\delta_{4}\right| T}{|\Omega|}\left\{\sum_{k=1}^{m}\left(M_{1}\left(t_{k}-t_{k-1}\right)+M_{3}+|\lambda| M_{2}\right)+M_{1}\left(T-t_{m}\right)\right\} \\
& +\sum_{k=1}^{m}\left(M_{1} \frac{\left(t_{k}-t_{k-1}\right)^{2}}{1+q_{k-1}}+\rho^{*}|\lambda|\left(t_{k}-t_{k-1}\right)+M_{2}\right)+\rho^{*}|\lambda|\left(T-t_{m}\right) \\
& +\sum_{k=1}^{m}\left(M_{1}\left(t_{k}-t_{k-1}\right)+M_{3}+|\lambda| M_{2}\right)\left(T-t_{k}\right)+M_{1} \frac{\left(T-t_{m}\right)^{2}}{1+q_{m}}:=\sigma .
\end{aligned}
$$

Hence, we deduce that $\|\mathcal{S} x\| \leq \sigma$.

Step 3: $\mathcal{S}$ maps bounded sets into equicontinuous sets of $P C(J, \mathbb{R})$.

Let $\tau_{1}, \tau_{2} \in J_{i}=\left(t_{i}, t_{i+1}\right]$ for some $i \in\{0,1,2, \ldots, m\}, \tau_{1}<\tau_{2}, B_{\rho^{*}}$ be a bounded set of $P C(J, \mathbb{R})$ as in Step 2 , and let $x \in B_{\rho^{*}}$. Then we have

$$
\begin{aligned}
& \left|(\mathcal{S} x)\left(\tau_{2}\right)-(\mathcal{S} x)\left(\tau_{1}\right)\right| \\
& \quad \leq \frac{\left|\delta_{2}\right|\left|\tau_{2}-\tau_{1}\right|}{|\Omega|}\left\{\sum _ { k = 1 } ^ { m } \left(\int_{t_{k-1}}^{t_{k}} \int_{t_{k-1}}^{s}|f(r, x(r))| d_{q_{k-1}} r d_{q_{k-1}} s\right.\right.
\end{aligned}
$$




$$
\begin{aligned}
& \left.+|\lambda| \int_{t_{k-1}}^{t_{k}}|x(s)| d_{q_{k-1}} s+\left|I_{k}\left(x\left(t_{k}\right)\right)\right|\right) \\
& +\sum_{k=1}^{m}\left(\int_{t_{k-1}}^{t_{k}}|f(s, x(s))| d_{q_{k-1}} s+\left|I_{k}^{*}\left(x\left(t_{k}\right)\right)\right|+|\lambda|\left|I_{k}\left(x\left(t_{k}\right)\right)\right|\right)\left(T-t_{k}\right) \\
& \left.+\int_{t_{m}}^{T} \int_{t_{m}}^{s}|f(r, x(r))| d_{q_{m}} r d_{q_{m}} s+|\lambda| \int_{t_{m}}^{T}|x(s)| d_{q_{m}} s\right\} \\
& +\frac{\left|\delta_{4}\right|\left|\tau_{2}-\tau_{1}\right|}{|\Omega|}\left\{\sum_{k=1}^{m}\left(\int_{t_{k-1}}^{t_{k}}|f(s, x(s))| d_{q_{k-1}} s+\left|I_{k}^{*}\left(x\left(t_{k}\right)\right)\right|+|\lambda|\left|I_{k}\left(x\left(t_{k}\right)\right)\right|\right)\right. \\
& \left.+\int_{t_{m}}^{T}|f(s, x(s))| d_{q_{m}} s\right\}+|\lambda|\left|\int_{t_{i}}^{\tau_{2}} x(s) d_{q_{i}} s-\int_{t_{i}}^{\tau_{1}} x(s) d_{q_{i}} s\right| \\
& +\left|\tau_{2}-\tau_{1}\right| \sum_{k=1}^{i}\left(\int_{t_{k-1}}^{t_{k}}|f(s, x(s))| d_{q_{k-1}} s+\left|I_{k}^{*}\left(x\left(t_{k}\right)\right)\right|+|\lambda|\left|I_{k}\left(x\left(t_{k}\right)\right)\right|\right) \\
& +\left|\int_{t_{i}}^{\tau_{2}} \int_{t_{i}}^{s} f(r, x(r)) d_{q_{i}} r d_{q_{i}} s-\int_{t_{i}}^{\tau_{1}} \int_{t_{i}}^{s} f(r, x(r)) d_{q_{i}} r d_{q_{i}} s\right| \\
& \leq \frac{\left|\delta_{2}\right|\left|\tau_{2}-\tau_{1}\right|}{|\Omega|}\left\{\sum_{k=1}^{m}\left(M_{1} \frac{\left(t_{k}-t_{k-1}\right)^{2}}{1+q_{k-1}}+\rho^{*}|\lambda|\left(t_{k}-t_{k-1}\right)+M_{2}\right)\right. \\
& \left.+\sum_{k=1}^{m}\left(M_{1}\left(t_{k}-t_{k-1}\right)+M_{3}+|\lambda| M_{2}\right)\left(T-t_{k}\right)+M_{1} \frac{\left(T-t_{m}\right)^{2}}{1+q_{m}}+\rho^{*}|\lambda|\left(T-t_{m}\right)\right\} \\
& +\frac{\left|\delta_{4}\right|\left|\tau_{2}-\tau_{1}\right|}{|\Omega|}\left\{\sum_{k=1}^{m}\left(M_{1}\left(t_{k}-t_{k-1}\right)+M_{3}+|\lambda| M_{2}\right)+M_{1}\left(T-t_{m}\right)\right\} \\
& +\left|\tau_{2}-\tau_{1}\right| \rho^{*}|\lambda|+\left|\tau_{2}-\tau_{1}\right| \sum_{k=1}^{i}\left(M_{1}\left(t_{k}-t_{k-1}\right)+M_{3}+|\lambda| M_{2}\right) \\
& +\left|\tau_{2}-\tau_{1}\right| M_{1} \frac{\left(\tau_{2}+\tau_{1}+2 t_{i}\right)}{1+q_{i}}
\end{aligned}
$$

The right-hand side of the above inequality is independent of $x$ and tends to zero as $\tau_{1} \rightarrow \tau_{2}$. As a consequence of Steps 1 to 3, together with the Arzelá-Ascoli theorem, we deduce that $\mathcal{S}: P C(J, \mathbb{R}) \rightarrow P C(J, \mathbb{R})$ is completely continuous.

Step 4: We show that the set

$$
E=\{x \in P C(J, \mathbb{R}): x=\kappa \mathcal{S} x \text { for some } 0<\kappa<1\}
$$

\section{is bounded.}

Let $x \in E$. Then $x(t)=\kappa(\mathcal{S} x)(t)$ for some $0<\kappa<1$. Thus, for each $t \in J$, we have

$$
\begin{aligned}
x(t)= & \kappa(\mathcal{S} x)(t) \\
= & \frac{\kappa\left(\delta_{1}+\delta_{2} t\right)}{\Omega}\left\{\sum _ { k = 1 } ^ { m } \left(\int_{t_{k-1}}^{t_{k}} \int_{t_{k-1}}^{s} f(r, x(r)) d_{q_{k-1}} r d_{q_{k-1}} s\right.\right. \\
& \left.-\lambda \int_{t_{k-1}}^{t_{k}} x(s) d_{q_{k-1}} s+I_{k}\left(x\left(t_{k}\right)\right)\right)
\end{aligned}
$$


Tariboon and Ntouyas Boundary Value Problems 2014, 2014:85

Page 15 of 19

http://www.boundaryvalueproblems.com/content/2014/1/85

$$
\begin{aligned}
& +\sum_{k=1}^{m}\left(\int_{t_{k-1}}^{t_{k}} f(s, x(s)) d_{q_{k-1}} s+I_{k}^{*}\left(x\left(t_{k}\right)\right)+\lambda I_{k}\left(x\left(t_{k}\right)\right)\right)\left(T-t_{k}\right) \\
& \left.+\int_{t_{m}}^{T} \int_{t_{m}}^{s} f(r, x(r)) d_{q_{m}} r d_{q_{m}} s-\lambda \int_{t_{m}}^{T} x(s) d_{q_{m}} s\right\} \\
& +\frac{\kappa\left(\delta_{3}+\delta_{4} t\right)}{\Omega}\left\{\sum_{k=1}^{m}\left(\int_{t_{k-1}}^{t_{k}} f(s, x(s)) d_{q_{k-1}} s+I_{k}^{*}\left(x\left(t_{k}\right)\right)+\lambda I_{k}\left(x\left(t_{k}\right)\right)\right)\right. \\
& \left.+\int_{t_{m}}^{T} f(s, x(s)) d_{q_{m}} s\right\}+\kappa \sum_{0<t_{k}<t}\left(\int_{t_{k-1}}^{t_{k}} \int_{t_{k-1}}^{s} f(r, x(r)) d_{q_{k-1}} r d_{q_{k-1}} s\right. \\
& \left.-\lambda \int_{t_{k-1}}^{t_{k}} x(s) d_{q_{k-1}} s+I_{k}\left(x\left(t_{k}\right)\right)\right) \\
& +\kappa \sum_{0<t_{k}<t}\left(\int_{t_{k-1}}^{t_{k}} f(s, x(s)) d_{q_{k-1}} s+I_{k}^{*}\left(x\left(t_{k}\right)\right)+\lambda I_{k}\left(x\left(t_{k}\right)\right)\right)\left(t-t_{k}\right) \\
& +\kappa \int_{t_{k}}^{t} \int_{t_{k}}^{s} f(r, x(r)) d_{q_{k}} r d_{q_{k}} s-\kappa \lambda \int_{t_{k}}^{t} x(s) d_{q_{k}} s .
\end{aligned}
$$

This implies by $\left(\mathrm{H}_{3}\right)$ and $\left(\mathrm{H}_{4}\right)$ that for each $t \in J$, we have

$$
\begin{aligned}
& \|x\| \leq \frac{\left|\delta_{1}\right|+\left|\delta_{2}\right| T}{|\Omega|}\left\{\sum _ { k = 1 } ^ { m } \left(\int_{t_{k-1}}^{t_{k}} \int_{t_{k-1}}^{s} M_{1} d_{q_{k-1}} r d_{q_{k-1}} s\right.\right. \\
& \left.+|\lambda| \int_{t_{k-1}}^{t_{k}}|x(s)| d_{q_{k-1}} s+M_{2}\right) \\
& +\sum_{k=1}^{m}\left(\int_{t_{k-1}}^{t_{k}} M_{1} d_{q_{k-1}} s+M_{3}+|\lambda| M_{2}\right)\left(T-t_{k}\right) \\
& \left.+\int_{t_{m}}^{T} \int_{t_{m}}^{s} M_{1} d_{q_{m}} r d_{q_{m}} s+|\lambda| \int_{t_{m}}^{T}|x(s)| d_{q_{m}} s\right\} \\
& +\frac{\left|\delta_{3}\right|+\left|\delta_{4}\right| T}{|\Omega|}\left\{\sum_{k=1}^{m}\left(\int_{t_{k-1}}^{t_{k}} M_{1} d_{q_{k-1}} s+M_{3}+|\lambda| M_{2}\right)+\int_{t_{m}}^{T} M_{1} d_{q_{m}} s\right\} \\
& +\sum_{k=1}^{m}\left(\int_{t_{k-1}}^{t_{k}} \int_{t_{k-1}}^{s} M_{1} d_{q_{k-1}} r d_{q_{k-1}} s+|\lambda| \int_{t_{k-1}}^{t_{k}}|x(s)| d_{q_{k-1}} s+M_{2}\right) \\
& +\sum_{k=1}^{m}\left(\int_{t_{k-1}}^{t_{k}} M_{1} d_{q_{k-1}} s+M_{3}+|\lambda| M_{2}\right)\left(T-t_{k}\right) \\
& +\int_{t_{m}}^{T} \int_{t_{m}}^{s} M_{1} d_{q_{m}} r d_{q_{m}} s+|\lambda| \int_{t_{m}}^{T}|x(s)| d_{q_{m}} s \\
& \leq \frac{\left|\delta_{1}\right|+\left|\delta_{2}\right| T+|\Omega|}{|\Omega|}\left\{M_{1} \sum_{k=1}^{m+1} \frac{\left(t_{k}-t_{k-1}\right)^{2}}{1+q_{k-1}}+|\lambda|\|x\| T+m M_{2}\right. \\
& \left.+\sum_{k=1}^{m}\left(M_{1}\left(t_{k}-t_{k-1}\right)+M_{3}+|\lambda| M_{2}\right)\left(T-t_{k}\right)\right\} \\
& +\frac{\left|\delta_{3}\right|+\left|\delta_{4}\right| T}{|\Omega|}\left\{M_{1} T+m M_{3}+m|\lambda| M_{2}\right\} .
\end{aligned}
$$


Setting

$$
\begin{aligned}
\Gamma= & \frac{\left|\delta_{1}\right|+\left|\delta_{2}\right| T+|\Omega|}{|\Omega|}\left\{M_{1} \sum_{k=1}^{m+1} \frac{\left(t_{k}-t_{k-1}\right)^{2}}{1+q_{k-1}}+m M_{2}\right. \\
& \left.+\sum_{k=1}^{m}\left(M_{1}\left(t_{k}-t_{k-1}\right)+M_{3}+|\lambda| M_{2}\right)\left(T-t_{k}\right)\right\} \\
& +\frac{\left|\delta_{3}\right|+\left|\delta_{4}\right| T}{|\Omega|}\left\{M_{1} T+m M_{3}+m|\lambda| M_{2}\right\},
\end{aligned}
$$

we have

$$
\|x\| \leq \frac{\left|\delta_{1}\right|+\left|\delta_{2}\right| T+|\Omega|}{|\Omega|}|\lambda|\|x\| T+\Gamma
$$

which yields

$$
\|x\| \leq \frac{\Gamma}{1-\frac{\left|\delta_{1}\right|+\left|\delta_{2}\right| T+|\Omega|}{|\Omega|}|\lambda| T}:=M .
$$

This shows that the set $E$ is bounded. As a consequence of Schaefer's fixed point theorem, we conclude that $\mathcal{S}$ has a fixed point which is a solution of the impulsive $q_{k}$-difference Langevin boundary value problem (1.4).

\section{Examples}

Example 4.1 Consider the following boundary value problem for the second-order impulsive $q_{k}$-difference Langevin equation:

$$
\left\{\begin{array}{l}
D_{\left(\frac{2 k+1}{5 k+2}\right) \frac{1}{2}}\left(D_{\left(\frac{2 k+1}{5 K+}\right)} \frac{1}{2}+\frac{1}{10}\right) x(t)=\frac{t}{e^{2 t}(10+t)^{2}} \cdot \frac{|x(t)|}{(1+|x(t)|)}, \quad t \in J=[0,1], t \neq t_{k}, \\
\Delta x\left(t_{k}\right)=\frac{\left|x\left(t_{k}\right)\right|}{9\left(9+\left|x\left(t_{k}\right)\right|\right)}, \quad t_{k}=\frac{k}{10}, k=1,2, \ldots, 9, \\
D_{\left(\frac{2 k+1}{5 k+2}\right)} x\left(t_{k}^{+}\right)-D_{\left(\frac{2 k-1}{5}\right) \frac{1}{2}} x\left(t_{k}\right)=\frac{1}{8} \tan ^{-1}\left(\frac{1}{10} x\left(t_{k}\right)\right), \quad t_{k}=\frac{k}{10}, k=1,2, \ldots, 9, \\
\frac{1}{7} x(0)+\frac{2}{9} D_{\frac{1}{\sqrt{2}}} x(0)=x(1), \quad \frac{2}{7} x(0)+\frac{1}{9} D_{\frac{1}{\sqrt{2}}} x(0)=D_{\sqrt{\frac{1}{47}}} x(1) .
\end{array}\right.
$$

Here $q_{k}=\sqrt{(2 k+1) /(5 k+2)}, k=0,1,2, \ldots, 9, m=9, T=1, \lambda=1 / 10, \alpha=1 / 7, \beta=2 / 9$, $\gamma=2 / 7, \eta=1 / 9, f(t, x)=(t|x(t)|) /\left(e^{2 t}(10+t)^{2}(1+|x(t)|)\right), I_{k}(x)=|x| /(9(9+|x|))$, and $I_{k}^{*}(x)=$ $(1 / 8) \tan ^{-1}(x / 10)$. Since

$$
\begin{aligned}
& |f(t, x)-f(t, y)| \leq(1 / 100)|x-y|, \\
& \left|I_{k}(x)-I_{k}(y)\right| \leq(1 / 81)|x-y| \text { and }\left|I_{k}^{*}(x)-I_{k}^{*}(y)\right| \leq(1 / 80)|x-y|,
\end{aligned}
$$

then $\left(\mathrm{H}_{1}\right)$ and $\left(\mathrm{H}_{2}\right)$ are satisfied with $L_{1}=(1 / 100), L_{2}=(1 / 81), L_{3}=(1 / 80)$. We can find that $\Omega=3,103 / 3,150, \delta_{1}=(-13) / 15, \delta_{2}=46 / 75, \delta_{3}=7 / 9, \delta_{4}=(-277) / 315$ and thus

$$
\Lambda_{1} \approx 0.920497882<1 .
$$

Hence, by Theorem 3.1, the boundary value problem (4.1) has a unique solution on $[0,1]$. 
Example 4.2 Consider the following boundary value problem for the second-order impulsive $q_{k}$-difference Langevin equation:

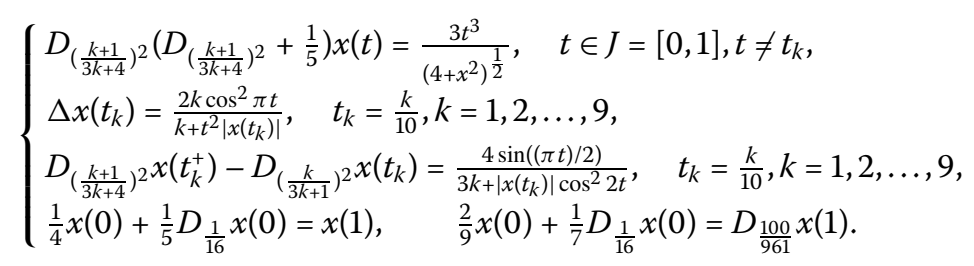

Here $q_{k}=((k+1) /(3 k+4))^{2}, k=0,1,2, \ldots, 9, m=9, T=1, \lambda=1 / 5, \alpha=1 / 4, \beta=1 / 5$, $\gamma=2 / 9, \eta=1 / 7, f(t, x)=\left(\left(3 t^{3}\right) /\left(4+x^{2}\right)^{1 / 2}\right), I_{k}(x)=\left(\left(2 k \cos ^{2} \pi t\right) /\left(k+t^{2}|x|\right)\right)$, and $I_{k}^{*}(x)=$ $\left((4 \sin ((\pi t) / 2)) /\left(3 k+|x| \cos ^{2} 2 t\right)\right)$. Clearly,

$$
|f(t, x)|=\left|\frac{3 t^{3}}{\left(4+x^{2}\right)^{\frac{1}{2}}}\right| \leq \frac{3}{2}, \quad\left|I_{k}(x)\right|=\left|\frac{2 k \cos ^{2} \pi t}{k+t^{2}|x|}\right| \leq 2
$$

and

$$
\left|I_{k}^{*}(x)\right|=\left|\frac{4 \sin ((\pi t) / 2)}{3 k+|x| \cos ^{2} 2 t}\right| \leq \frac{4}{3} .
$$

We can find that

$$
\frac{\left|\delta_{1}\right|+\left|\delta_{2}\right| T+|\Omega|}{|\Omega|}|\lambda| T=\frac{13,958}{26,273}<1,
$$

where $\Omega=(\alpha-1)(\eta-1)-\lambda T(\eta+\beta \lambda-\alpha)+\gamma(T-\beta)=26,273 / 31,500, \delta_{1}=\eta-1+\beta \lambda=$ $(-143) / 175$ and $\delta_{2}=\lambda(\eta+\beta \lambda-1-\alpha)+1-\gamma=17,777 / 31,500$.

Hence, by Theorem 3.2, the boundary value problem (4.2) has at least one solution on $[0,1]$.

\section{Competing interests}

The authors declare that they have no competing interests.

\section{Authors' contributions}

Both authors contributed equally in this article. They read and approved the final manuscript.

\section{Author details}

${ }^{1}$ Nonlinear Dynamic Analysis Research Center, Department of Mathematics, Faculty of Applied Science, King Mongkut's University of Technology North Bangkok, Bangkok, Thailand. 2Department of Mathematics, University of loannina, loannina, 451 10, Greece.

\section{Authors' information}

Sotiris K Ntouyas is a member of Nonlinear Analysis and Applied Mathematics (NAAM) - Research Group at King Abdulaziz University, Jeddah, Saudi Arabia.

\section{Acknowledgements}

We would like to thank the reviewers for their valuable comments and suggestions on the manuscript. The research of J Tariboon is supported by King Mongkut's University of Technology North Bangkok, Thailand.

\section{Received: 11 November 2013 Accepted: 7 April 2014 Published: 02 May 2014}

\section{References}

1. Samko, SG, Kilbas, AA, Marichev, Ol: Fractional Integrals and Derivatives: Theory and Applications. Gordon \& Breach, Yverdon (1993)

2. Podlubny, I: Fractional Differential Equations. Academic Press, San Diego (1999) 
3. Kilbas, AA, Srivastava, HM, Trujillo, JJ: Theory and Applications of Fractional Differential Equations. North-Holland Mathematics Studies, vol. 204. Elsevier, Amsterdam (2006)

4. Sabatier, J, Agrawal, OP, Machado, JAT (eds.): Advances in Fractional Calculus: Theoretical Developments and Applications in Physics and Engineering. Springer, Dordrecht (2007)

5. Lakshmikantham, V, Leela, S, Vasundhara Devi, J: Theory of Fractional Dynamic Systems. Cambridge Academic Publishers, Cambridge (2009)

6. Baleanu, D, Golmankhaneh, AK, Golmankhaneh, AK: Fractional Nambu mechanics. Int. J. Theor. Phys. 48, 1044-1052 (2009)

7. Ahmad, B, Ntouyas, SK: Nonlinear fractional differential equations and inclusions of arbitrary order and multi-strip boundary conditions. Electron. J. Differ. Equ. 2012, 98 (2012)

8. Ahmad, B, Nieto, JJ: Sequential fractional differential equations with three-point boundary conditions. Comput. Math. Appl. 64, 3046-3052 (2012)

9. Ahmad, B, Otero-Espinar, V: Existence of solutions for fractional differential inclusions with anti-periodic boundary conditions. Bound. Value Probl. 2009, Article ID 625347 (2009)

10. Chang, Y-K, Nieto, JJ: Some new existence results for fractional differential inclusions with boundary conditions. Math. Comput. Model. 49, 605-609 (2009)

11. Cernea, A: On the existence of solutions for nonconvex fractional hyperbolic differential inclusions. Commun. Math. Anal. 9(1), 109-120 (2010)

12. Ahmad, B: Existence results for fractional differential inclusions with separated boundary conditions. Bull. Korean Math. Soc. 47, 805-813 (2010)

13. Ahmad, B, Ntouyas, SK: Some existence results for boundary value problems fractional differential inclusions with non-separated boundary conditions. Electron. J. Qual. Theory Differ. Equ. 2010, 71 (2010)

14. Ahmad, B, Ntouyas, SK: A four-point nonlocal integral boundary value problem for fractional differential equations of arbitrary order. Electron. J. Qual. Theory Differ. Equ. 2011, 22 (2011)

15. Ahmad, B, Ntouyas, SK: Boundary value problems for q-difference inclusions. Abstr. Appl. Anal. 2011, Article ID $292860(2011)$

16. Ahmad, B, Nieto, J: Solvability of nonlinear Langevin equation involving two fractional orders with Dirichlet boundary conditions. Int. J. Differ. Equ. 2010, Article ID 649486 (2010)

17. Agarwal, RP, Ahmad, B: Existence theory for anti-periodic boundary value problems of fractional differential equations and inclusions. Comput. Math. Appl. 62, 1200-1214 (2011)

18. Sudsutad, W, Tariboon, J: Existence results of fractional integro-differential equations with $m$-point multi-term fractional order integral boundary conditions. Bound. Value Probl. 2012, 94 (2012)

19. Coffey, WT, Kalmykov, YP, Waldron, JT: The Langevin Equation, 2nd edn. World Scientific, Singapore (2004)

20. Lim, SC, Li, M, Teo, LP: Langevin equation with two fractional orders. Phys. Lett. A 372, 6309-6320 (2008)

21. Lim, SC, Teo, LP: The fractional oscillator process with two indices. J. Phys. A, Math. Theor. 42, Article ID 065208 (2009)

22. Uranagase, M, Munakata, T: Generalized Langevin equation revisited: mechanical random force and self-consistent structure. J. Phys. A, Math. Theor. 43, Article ID 455003 (2010)

23. Denisov, SI, Kantz, H, Hänggi, P: Langevin equation with super-heavy-tailed noise. J. Phys. A, Math. Theor. 43, Article ID 285004 (2010)

24. Lozinski, A, Owens, RG, Phillips, TN: The Langevin and Fokker-Planck equations in polymer rheology. In: Handbook of Numerical Analysis, vol. 16, pp. 211-303 (2011)

25. Lizana, L, Ambjörnsson, T, Taloni, A, Barkai, E, Lomholt, MA: Foundation of fractional Langevin equation: harmonization of a many-body problem. Phys. Rev. E 81, Article ID 051118 (2010)

26. Ahmad, B, Eloe, PW: A nonlocal boundary value problem for a nonlinear fractional differential equation with two indices. Commun. Appl. Nonlinear Anal. 17, 69-80 (2010)

27. Ahmad, B, Nieto, JJ, Alsaedi, A, El-Shahed, M: A study of nonlinear Langevin equation involving two fractional orders in different intervals. Nonlinear Anal., Real World Appl. 13, 599-606 (2012)

28. Kac, V, Cheung, P: Quantum Calculus. Springer, New York (2002)

29. Bangerezako, G: Variational q-calculus. J. Math. Anal. Appl. 289, 650-665 (2004)

30. Dobrogowska, A, Odzijewicz, A: Second order q-difference equations solvable by factorization method. J. Comput. Appl. Math. 193, 319-346 (2006)

31. Gasper, G, Rahman, M: Some systems of multivariable orthogonal q-Racah polynomials. Ramanujan J. 13, 389-405 (2007)

32. Ismail, MEH, Simeonov, P: q-Difference operators for orthogonal polynomials. J. Comput. Appl. Math. 233, 749-761 (2009)

33. Bohner, M, Guseinov, GS: The $h$-Laplace and $q$-Laplace transforms.J. Math. Anal. Appl. 365, 75-92 (2010)

34. El-Shahed, M, Hassan, HA: Positive solutions of $q$-difference equation. Proc. Am. Math. Soc. 138, 1733-1738 (2010)

35. Ahmad, B: Boundary-value problems for nonlinear third-order q-difference equations. Electron. J. Differ. Equ. 2011, 94 (2011)

36. Ahmad, B, Alsaedi, A, Ntouyas, SK: A study of second-order q-difference equations with boundary conditions. Adv. Differ. Equ. 2012, 35 (2012)

37. Ahmad, B, Ntouyas, SK, Purnaras, IK: Existence results for nonlinear q-difference equations with nonlocal boundary conditions. Commun. Appl. Nonlinear Anal. 19, 59-72 (2012)

38. Ahmad, B, Nieto, Jj: On nonlocal boundary value problems of nonlinear $q$-difference equations. Adv. Differ. Equ. 2012, 81 (2012)

39. Ahmad, B, Ntouyas, SK: Boundary value problems for q-difference inclusions. Abstr. Appl. Anal. 2011, Article ID $292860(2011)$

40. Zhou, W, Liu, H: Existence solutions for boundary value problem of nonlinear fractional q-difference equations. Adv. Differ. Equ. 2013, 113 (2013)

41. Yu, C, Wang, J: Existence of solutions for nonlinear second-order $q$-difference equations with first-order $q$-derivatives Adv. Differ. Equ. 2013, 124 (2013)

42. Lakshmikantham, V, Bainov, DD, Simeonov, PS: Theory of Impulsive Differential Equations. World Scientific, Singapore (1989) 
43. Samoilenko, AM, Perestyuk, NA: Impulsive Differential Equations. World Scientific, Singapore (1995)

44. Benchohra, M, Henderson, J, Ntouyas, SK: Impulsive Differential Equations and Inclusions, vol. 2. Hindawi Publishing Corporation, New York (2006)

45. Tariboon, J, Ntouyas, SK: Quantum calculus on finite intervals and applications to impulsive difference equations. Adv. Differ. Equ. 2013, 282 (2013)

10.1186/1687-2770-2014-85

Cite this article as: Tariboon and Ntouyas: Nonlinear second-order impulsive $q$-difference Langevin equation with boundary conditions. Boundary Value Problems 2014, 2014:85

Submit your manuscript to a SpringerOpen ${ }^{\circ}$ journal and benefit from:

- Convenient online submission

- Rigorous peer review

- Immediate publication on acceptance

- Open access: articles freely available online

- High visibility within the field

- Retaining the copyright to your article

Submit your next manuscript at $>$ springeropen.com 